SHORT REPORT

\title{
Ataxic arm movements after thalamotomy for Parkinsonian tremor
}

\author{
T E Kimber, B P Brophy, P D Thompson
}

J Neurol Neurosurg Psychiatry 2003;74:258-259

Voluntary finger-nose movements of the arm were analysed in six patients undergoing stereotaxic nucleus ventralis intermedius thalamotomy for relief of severe Parkinsonian tremor. In all cases thalamotomy acutely abolished tremor in the contralateral arm. In the early postoperative phase, ataxia of the arm contralateral to the operated side was also seen. Ataxia was transient, lasting between 7 and 21 days postoperatively. This observation suggests that a lesion of the Vim nucleus interrupts cerebellar input to the thalamus, and supports the concept that abnormal cerebellar activity is an important contributor to the generation of tremor in Parkinson's disease.

$\mathrm{P}$ arkinsonian tremor is thought to be sustained by oscillatory activity in neuronal circuits linking the ventral lateral thalamic nuclei and the motor cortex. ${ }^{12}$ Some neurones in ventralis oralis posterior (Vop, which receives input from the pallidum) and ventralis intermedius (Vim, which receives input from the cerebellum) display rhythmic activity related to tremor at frequencies of 4-6 $\mathrm{Hz}^{3-5}$ corresponding to the frequency of parkinsonian tremor. The precise relation of the rhythmic thalamic discharges and central oscillatory circuits remains unclear, though rhythmic thalamic activity does not appear to be driven by peripheral sensory feedback or efferent copy from the motor cortex. ${ }^{136-8}$ Stereotaxic lesions of Vim and Vop abolish Parkinsonian tremor in the contralateral arm in $80-90 \%$ of cases. The precise mechanisms responsible for tremor and the abolition of tremor after Vim thalamotomy remain the subject of debate. In particular, it is not known whether the critical lesion for abolition of tremor interrupts cerebellar or pallidal inputs to the thalamus or the neuronal targets of these inputs in either Vop or Vim. The observation of postoperative ataxia led to the prospective examination of a series of patients undergoing Vim thalamotomy to see if this was a consistent finding and whether it correlated in any way with the surgical outcome.

\section{METHODS}

\section{Subjects}

We studied six consecutive patients undergoing Vim stereotaxic thalamotomy for severe Parkinsonian tremor refractory to medical treatment. The subjects (three men and three women) were aged between 55 and 75 years.

\section{Thalamotomy}

After attachment of the Leksell stereotaxic frame, computed tomography of the brain was undertaken to measure the three dimensional coordinates of the anterior and posterior commissures (AC, PC). The Vim nucleus was located using the construct of Guiot, $6 \mathrm{~mm}$ anterior to the PC, $2 \mathrm{~mm}$ above the plane of the AC-PC line, and approximately $11 \mathrm{~mm}$ lateral to the wall of the third ventricle. A monopolar electrode with a
$2 \mathrm{~mm}$ long exposed tip was introduced into the target under local anaesthetic. The target was confirmed by noting the responses to electrical stimulation through the electrode. Pacing and disruption of tremor were observed at low frequency stimulation, and asterixis of the contralateral arm, paraesthesiae of the contralateral arm and face, and reduction of tremor were observed at high frequency stimulation. Lesions were then made by heating the electrode tip to between $70^{\circ} \mathrm{C}$ and $76^{\circ} \mathrm{C}$. Postoperative confirmation of lesion location was obtained in all cases.

\section{Analysis of unrestrained arm movements}

Subjects underwent routine neurological examination and were videotaped performing a "finger-nose" arm movement before and after thalamotomy. The arm movement was unrestrained and based on the standard neurological examination of placing the index finger on the nose, then moving to the target and back to the nose. Frame by frame analysis of the videotapes was undertaken to trace the trajectory of the index finger during this task. Subjects were examined on three occasions: preoperatively, one or two days postoperatively, and between one and two months postoperatively.

\section{RESULTS}

All six subjects had complete abolition of Parkinsonian tremor in the contralateral arm after Vim thalamotomy. In the first few postoperative days, all showed signs of past pointing and terminal intention tremor of the arm during the finger-nose movement (fig 1). Mild hypotonia of the limb was also evident on clinical examination. Two patients had mild ataxia of gait and the contralateral leg. The upper limb ataxia was asymptomatic in five of the six patients. No weakness, reflex change, or sensory impairment were evident in the limb contralateral to the thalamotomy, and no visual impairment occurred postoperatively. By the one to two months follow up, the ataxia had resolved in all but one patient. In that case, the ataxia in the immediate postoperative period was symptomatic.

\section{DISCUSSION}

Limb ataxia with past pointing and intention tremor, of varying degree, was a consistent postoperative finding after stereotaxic Vim thalamotomy for Parkinsonian tremor in the patients in this series. In all patients, ataxia accompanied successful abolition of limb tremor. The ataxia could not be accounted for by weakness, deafferentation or sensory loss, or visual field defects. The ataxic arm movements and hypotonia had the characteristics of cerebellar ataxia after an acute cerebellar injury. In the majority of patients in our study, contralateral limb ataxia resolved over the month following surgery. Transient limb ataxia after thalamotomy has been noted infrequently in previous studies of stereotaxic surgery for parkinsonian tremor. ${ }^{9-14}$

There is evidence implicating the cerebellum in the pathogenesis of tremor of various types. Essential tremor may 


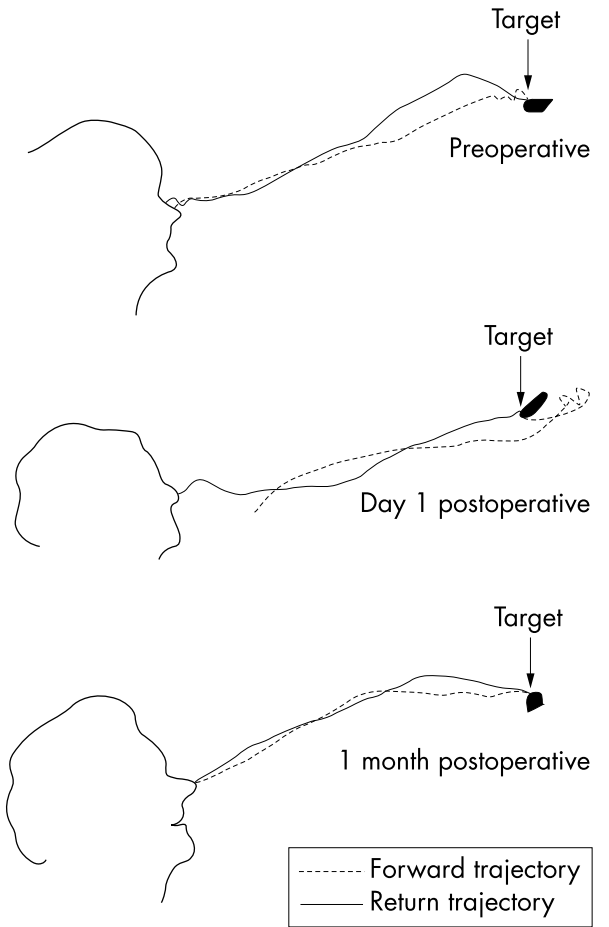

Figure 1 Trace of finger trajectory during a movement of the arm from the nose to a target in a patient with severe parkinsonian tremor before thalamotomy (upper panel), the day following surgery (middle panel), and one month after surgery. In the upper panel a terminal postural tremor is seen in both directions of movement. In the immediate postoperative period there was past pointing with terminal corrective movements; this had settled when the patient was re-examined one month later.

be abolished after ipsilateral cerebellar stroke or injury. ${ }^{15}$ Cerebellar synaptic activity on positron emission tomography (PET) is increased in subjects with essential tremor compared with normal subjects. ${ }^{16}$ Moreover, in patients with Parkinson's disease, stimulation within the Vim nucleus at intensities sufficient to abolish tremor causes a reduction in cerebellar synaptic activity as measured by PET, whereas there was no change following stimulation at intensities that do not abolish tremor. ${ }^{17}$ This effect was observed in both medial cerebellar structures, and so could not be attributed to a reduction of proprioceptive input to one cerebellar hemisphere following cessation of tremor. The investigators concluded that high frequency Vim stimulation led to inhibition of a central oscillatory mechanism. ${ }^{17}$ To these observations we add the finding that successful abolition of tremor by Vim thalamotomy is accompanied by transient cerebellar ataxia, suggesting that the surgical lesion interrupts cerebellar projections to Vim.

We cannot exclude the possibility that abolition of tremor is related to inactivation of Vop neurones or pallidal afferents adjacent to Vim, and that transient ataxia is caused by interruption of cerebellar afferents to Vim, either directly or by perioperative oedema. These explanations, however, would not account for the physiological studies discussed above implicating the cerebellum in the genesis of tremor. Resolution of the limb ataxia over the weeks following thalamotomy is consistent with adaptation and compensation for the acute cerebellar deficit, as is well described after cerebellar injury. ${ }^{18}$

\section{Conclusions}

The most parsimonious explanation for post-thalamotomy ataxia is interruption of cerebellothalamic connections. The implications of this finding fit well with recent observations on the pathophysiological basis of tremor and reinforce the notion that the cerebellum and cerebellothalamic connections are critical links in the oscillatory motor circuits responsible for Parkinsonian tremor.

\section{Authors' affiliations}

T E Kimber, P D Thompson, University Department of Medicine and Department of Neurology, Royal Adelaide Hospital and University of Adelaide, Adelaide, Australia

B P Brophy, Department of Neurosurgery, Royal Adelaide Hospital

Competing interests: none declared

Correspondence to: Professor P D Thompson, University Department of Medicine, Royal Adelaide Hospital, North Terrace, Adelaide, South

Australia 5000; philip.thompson@adelaide.edu.au

Received 9 July 2001

In revised form 12 August 2002

Accepted 23 October 2002

\section{REFERENCES}

1 Lamarre $\mathrm{Y}$, Joffroy AJ. Experimental tremor in monkey: activity of thalamic on precentral neurons in the absence of peripheral feedback. Adv Neurol 1979;24:109-22.

2 Volkmann J, Joliot M, Mogilner A, et al. Central motor loop oscillations in parkinsonian resting tremor revealed by magnetoencephalogaphy. Neurology 1996;46:1359-70.

3 Jasper HH, Bertrand $\mathrm{G}$. Thalamic units involved in somatic sensation and voluntary and involuntary movements in man. In: Purpura DP, Yahr MD, eds. The thalamus. New York: Columbia University Press, 1966:365-90.

4 Albe-Fessard D, Guiot G, Lamarre Y, et al. Activation of thalamocortical projections related to tremorogenic processes. Purpura DP, Yahr MD, eds. The thalamus. New York: Columbia University Press, 1966:237-53.

5 Ohye C, Albe-Fessard D. Rhythmic discharges related to tremor in humans and monkeys. In: Chalazonitis N, Boisson M, eds. Abnormal neuronal discharges. New York: Raven Press, 1978:37-48.

6 Ohye C, Saito Y, Fukamachi A, et al. An analysis of the rhythmic and non-rhythmic burst discharges in the human thalamus. J Neurol Sci 1974;22:245-59.

7 Velasco F, Molina-Negro P. Electrophysiological topography of the human diencephalon. J Neurosurg 1973;38:204-14.

8 Lenz FA, Kwan HC, Martin RL, et al. Single unit analysis of the human ventral thalamic nuclear group. Tremor-related activity in functionally identified cells. Brain 1994;117:531-4.

9 Markham CH, Rand RW. Stereotactic surgery in Parkinson's disease. Arch Neurol 1963;8:621-31.

10 Selby G. Stereotaxic surgery for the relief of Parkinson's disease. Part 2. An analysis of the results in a series of 303 patients (413 operations). J Neurol Sci 1967;5:343-75.

11 Hoehn MM, Yahr MD. Evaluation of long term results of surgical therapy. In: Gillingham FJ, Donaldson IML, eds. Third symposium on Parkinson's disease. Edinburgh: Livingstone, 1969:274-80.

12 Yoshida M, Okada K, Watanabe M, et al. Analysis of tremulous movements after thalamotomy correlated to intrathalamic therapeutic lesions. Appl Neurophysiol 1976/77;39:311-14.

13 Yasui N, Narabayashi H, Kondo T, et al. Slight cerebellar signs in stereotactic thalamotomy and subthalamotomy for Parkinsonism. Appl Neurophysiol 1976/77;39:315-20.

14 Jankovic J, Cardoso F, Grossman RG, et al. Outcome after stereotactic thalamotomy for parkinsonian, essential and other types of tremor. Neurosurgery 1995;37:680-6.

15 Dupuis MJM, Delwaide PJ, Boucquey, et al. Homolateral disappearance of essential tremor after cerebellar stroke. Mov Disord 1989:4:183-7.

16 Jenkins IH, Bain PG, Colebatch JG, et al. A positron emission tomography study of essential tremor: evidence for overactivity of cerebellar connections. Ann Neurol 1993;34:82-90.

17 Deiber M, Pollak P, Passingham R, et al. Thalamic stimulation and suppression of parkinsonian tremor: evidence of a cerebellar deactivation using positron emission tomography. Brain 1993;1 16:267-79.

18 Martin TA, Keating JG, Goodkin HP, et al. Throwing while looking through prisms. I. Focal cerebellar lesions impair adaptation. Brain 1996;119:1183-98. 